\title{
Study of Management of anemia in Chronic Kidney Disease Patients
}

\author{
Meby Susan Mathew, Nama Ravi Sneha Keerthi, Neelathahalli Kasturirangan Meera*
}

Meera N.K, Visveswarapura Institute of Pharmaceutical Sciences, $22^{\text {nd }}$ Main, $24^{\text {th }}$ Cross, Opposite to BDA Complex, BSK $2^{\text {nd }}$ Stage, Bengaluru-560070, Karnataka, INDIA.

\begin{abstract}
Background: Anemia is the common comorbidity in patients with chronic kidney disease (CKD). Clinical studies have shown that erythropoietin-stimulating agents decrease the need for transfusions and improve the quality of life. Objectives: The objectives were to assess the management of anemia, provide counseling and to create awareness among the patients using patient information leaflets. Methodology: A prospective, hospital based study was conducted for a period of nine months on 82 dialysis patients suffering from anemia in chronic renal failure condition. A written informed consent was obtained from all the patients before inclusion in the study. Patient demographics, laboratory data and treatment chart were reviewed. Patient information leaflets, diet chart were given along with counseling at base line and during follow ups. The study results were analyzed by descriptive statistical analysis. Student t test was used to find significance of study parameters at base line and follow up. Results: The mean hemoglobin levels showed an increase in patients with epoetin therapy. The parameters like serum ferritin, serum iron, TSAT and serum folate showed an increase in both patients who received epoetin as well as those on oral iron therapy. Conclusion: It can be concluded that early detection and treatment of anemia with effective counseling from clinical pharmacist benefits CKD patients.
\end{abstract}

key words: Chronic kidney disease, Erythropoietin, Hemodialysis, Anemia

\section{INTRODUCTION}

Anemia in patients with chronic kidney disease (CKD) is multi factorial in origin, primarily associated with relative erythropoietin production deficiency as the glomerular filtration rate (GFR) falls. The evidence now seems to suggest that hemoglobin target levels between $10-11.5 \mathrm{~g} / \mathrm{dL}$ should be the aim for patients with CKD. ${ }^{1}$

The epidemiology of CKD patients with diabetes are at a greater risk of developing anemia earlier in the course of the disease (associated with inappropriately low levels of erythropoietin). Comparing patients with similar glomerular filtration rate (eGFR) and erythropoietin levels, those with type 2 diabetes are generally more anemic. ${ }^{2}$

Anemia can be determined using simple blood tests which gives values for hematocrit (Hct) and hemoglobin (Hb), RBC indices, Reticulocyte count, Iron parameters like
Serum iron, Total Iron binding capacity, Percent transfer in saturation and serum ferrit in and test for occult blood in stool. The National Kidney Foundation's Dialysis Outcome Quality Initiative (K/DOQI) guideline recommends that the cause of anemia be determined, monitored and treated.

Patients with moderate-to-severe CKD may DOI: 10.5530/ijopp.9.3.6

Address for correspondence: Dr. Neelathahalli Kasturirangan Meera .

N.K, Visveswarapura Institute of Pharmaceutical Sciences, 22nd Main, 24th Cross, Opposite to BDA Complex, BSK 2nd Stage,

Bengaluru-560070,

Karnataka, INDIA.

Ph. No: +91 9844315714

Email id: meera_satish@ yahoo.com

require more frequent $\mathrm{Hb}$ monitoring since the likelihood of anemia is greater in this population; more frequent monitoring (at least monthly) is also required during treatment with stimulants of erythropoiesis and during and after acute episodes of uncontrolled comorbid disease like poor glycemic control.

Treatment in patients with CKD- associated anemia involves, correction of iron

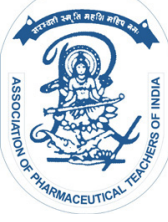

www.ijopp.org 
deficiency with iron supplementation that is intended to assure adequate iron stores for erythropoiesis, correct iron deficiency, and, in patients receiving erythropoietin stimulating agents(ESA) treatment, prevent development of iron deficiency. Iron supplementation, particularly with intravenous iron, can enhance erythropoiesis and raise $\mathrm{Hb}$ levels in $\mathrm{CKD}$ patients with anemia even when TSAT and ferrit in levels are not indicative of absolute iron deficiency, and even when bone marrow studies reveal adequate iron stores. Iron treatment, particularly when administered intravenously, has also been consistently demonstrated to improve the erythropoietic response to ESA treatment.

Decisions about the preferred route of iron supplementation (oral or intravenous, IV therapy) should take into consideration severity of anemia and iron deficiency, the response, tolerance and adherence to prior oral iron administration, costs, and ease of obtaining venous access balanced against the desire to preserve venous access sites.

Oral iron is prescribed to provide approximately $200 \mathrm{mg}$ of elemental iron daily. IV iron administration can lead to a greater increase in $\mathrm{Hb}$ concentration, a lower ESA dose, or both.

IV iron may be provided as a single large dose or as repeated smaller doses depending on the specific IV iron preparation used and common practice to provide an initial course of IV iron amounting to approximately 1000mg; this may be repeated if an initial dose fails to increase $\mathrm{Hb}$ level and/or allow a decrease in ESA dose and if the TSAT remains $\leq 30 \%$ and serum ferritin remains $\leq 500 \mathrm{ng} / \mathrm{ml}(\leq 500 \mathrm{mg} / \mathrm{l}){ }^{3}$

Treatment with erythropoiesis-stimulating agents should be offered to patients with anemia of CKD who are likely to benefit in terms of quality of life and physical function. One study reported that darbepoet in Alfa weekly or every two weeks was more efficient in achieving target hemoglobin than those on weekly epoetin alfa, with fewer dose changes and minor vascular access complications. The time taken for erythropoietin treatment to be effective will depend on individual patient factors, such as degree of anemia, degree of kidney disease and presence of other adverse factors- eg iron deficiency. ${ }^{2}$

Where possible, blood transfusions should be avoided in patients in whom kidney transplant is a treatment option. $^{2}$

This study was aimed at assessing anemia management in CKD patients and to create awareness regarding medication adherence and life style changes.

\section{METHODOLOGY}

This was a hospital based prospective study conducted in the dialysis department of a tertiary care teaching hospital, Bangalore, for a period of 9 months. A total of 82 patients from the dialysis centre at the hospital (both inpatients and outpatients) who satisfied the study criteria and consented to participate in this study were included in the study. The complete project was done after obtaining the permission grated by the ethical committee of VIPS, Bangalore.

\section{Study criteria}

- Inclusion Criteria

CKD patients having anemia.

CKD patients undergoing hemodialysis.

CKD patients having a negative result for the laboratory test for occult blood in stool.

\section{- Exclusion Criteria}

Patients undergoing peritoneal dialysis and having GI bleed.

- Sources of data:

Patient data relevant to the study was obtained from the following sources;

Direct patient and their care-givers interview

Medication chart review

Patient case history

Follow-up note

\section{Study Procedure}

The study was conducted on 82 patients who were suffering from anemia in CKD and were on dialysis. Patients who had not given their consent were excluded from the study.

The patient pro forma to collect information about patient's demographic details and laboratory data was prepared. During the study-patient information leaflets and a general diet chart were prepared in consultation with dietician an appropriate diet chart was prepared for the CKD patients to follow.

\section{Methods of data collection and study of management of anemia}

Prescriptions and dialysis booklets of the outpatients and treatment charts of the inpatients were reviewed prospectively and details like patients age, gender, occupation, educational background, and laboratory data which included the patient's pre-iron levels, TIBC, TSAT and serum folate levels, serum creatinine levels, serum iron levels, TIBC, TSAT were collected and follow up was conducted for the study management of anemic CKD patients enrolled in the study. 


\section{Patient Counseling}

Counseling was provided to all patients suffering from anemia in CKD and on dialysis. Counseling was done in the local language and we used the counseling tips provided by the national kidney foundation as the reference. Patient counseling included counseling on the disease, medication, diet, exercise. Patient information leaflet designed as part of the study was given to each patient.

\section{Statistics}

Descriptive statistical analysis has been carried out in the present study. Results on continuous measurements are presented on Mean \pm SD (Min-Max) and results on categorical measurements are presented in Number (\%). The statistical software namely SPSS 15.0, Stata 8.0, MedCalc 9.0.1 and Systat 11.0 were used for the analysis of the data and Microsoft word and Excel have been used to generate graphs, tables etc.

\section{RESULTS AND DISCUSSION}

The present study included 82 patients as the total number of patients, out of which 23 were females and 59 males. In our study we found that majority of the patients belonged to the age group of 51-60 years and $41-50$ years (35.4\% and $25.6 \%$ respectively) showing that older people are more vulnerable to chronic kidney disease. Table 1 summarizes the distribution of the study patients with respect to gender and co-morbidity.

Rebecca J Schmidt has stated in her study that untreated anemia of chronic kidney disease is strongly associated with cardiovascular and renal complications, resulting in increased hospitalization and mortality. Our study also attempted to classify the patients based on their gender to evaluate and compare the mean values between both the groups. The patients were classified based on their co-morbid conditions and we found that all the patients who were included in the study had hypertension (100\%) while 33 patients of the total number of patients had diabetes mellitus, while left ventricular hypertrophy was present in 12 patients and angina pectoris in 4 patients, followed by congestive cardiac failure in 2 patients.

Rebecca J Schmidt also stated that monthly follow-ups is required to evaluate cardio renal health and to ensure that the hemoglobin levels do not overshoot the optimal range of $11-12 \mathrm{~g} / \mathrm{dL}$. Our study depicts the mean values of the laboratory tests such as serum creatinine, blood urea nitrogen and hemoglobin levels calculated for each month of the study, however tests like serum ferritin, TIBC, TSAT, serum iron and serum folate levels were carried out only two times during the entire study period probably due to patient's convenience and consent. ${ }^{4}$
Table 2 represents the mean levels of iron and folate parameters evaluated.

It was observed that laboratory tests such as serum ferritin, serum iron, total iron binding capacity (TIBC), and serum folate were carried out two times in a period of 9 months. The serum ferritin levels did increase for both males and females during the study period. There was a statistically significant difference in their mean ferritin levels in the first test where females had a higher ferritin level. Females showed a better mean serum ferritin level in the second test too when compared to the males, although the $\%$ improvement was greater for the males.

Serum iron levels were found to increase for both the categories, with males showing increase in both mean serum iron levels as well as \% improvement during the study period. Mean TIBC levels were also found to increase in both males and females which was however not statistically significant. Serum folate levels were also found to increase in both groups but to a greater extent in males than in females.

When the hemoglobin values of the patient were evaluated based on their gender it was found that the mean hemoglobin values increased every month when compared to the previous month and females showed between hemoglobin values when compared with males which was however not statistically significant.

The present study also classified the patients based on the treatment given for anemia as patients given erythropoietin and those not given erythropoietin. It was observed that there were only nine patients who were administered erythropoietin and the rest i.e. 73 patients were not administered erythropoietin.

Types of erythropoietin hormones and IV iron and oral iron administered are shown in table 3.

It was also found that there were 2 patients out of the total 82 patients who did not receive either erythropoietin or blood infusions because their $\mathrm{Hb}$ levels were greater than 10 and were therefore maintained on oral iron therapy.

A.K. Singh has stated in his study that significantly higher transferring saturation but not ferritin or soluble transferring receptor levels predicted a greater response; however their influence was not clinically significant in the study. Hence they concluded that none of the studied markers is a good predictor of response to anemia treatment in hemodialysis patients. ${ }^{5}$

During our study it was observed that serum ferritin, serum iron, TIBC, TSAT and serum folate values have shown an increase in their mean values in the second test when compared with their values in the first test which was done during the study period TSAT and 
serum iron showed statistically significant increase in their mean values.

Our study observed that patients having hemoglobin levels $<6$ were given blood transfusions followed by oral iron tablets, however patients with hemoglobin levels ranging from 6-10 were given either erythropoietin with IV iron or blood transfusions followed by oral iron tablets (200mg twice daily) based on their affordability and the patients with hemoglobin levels $>10$ were maintained on oral iron tablets (200mg once daily). According to Walter H. Horl, adult hemodialysis patients intravenous iron prevents iron restricted erythropoiesis during ESA therapy. ${ }^{6}$

There were 2 patients with hemoglobin levels less than 6 and $97.2 \%$ of the patients had their hemoglobin levels between 6-10 as well as 2 patients having hemoglobin level greater than or equal to 10 who were not given either blood transfusion or epoetin therapy. However in the month of January there were $5.4 \%$ of the patients with hemoglobin levels greater than or equal to 10 and $94.6 \%$ of the patients in the ranges of $6-10$, with no patients having hemoglobin level less than 6. Francesco Locatelli in his study stated that nowadays there are three erythropoieisis stimulating agents available in the market for the treatment of renal anemia: epoetin alfa, epoetin beta, darbapoetin alfa. Epoetin alfa and epoetin beta are both synthesized from Chinese hamster ovary cells both differing in the manufacturing processes between the two glycoproteins which reflect the differences in their carbohydrate moieties. A randomized crossover study was conducted on healthy volunteers, in which the terminal elimination half-life of intravenous epoetin beta was found to be $20 \%$ longer than that observed with intravenous epoetin alfa.?

Epoetin alpha which was given to 8 patients and epoetin beta which was given only to one patient were the types of epoetin administered to patients. IV iron administered included Ferric ammonium citrate and iron hydroxide polymatose comlex, similarly oral iron prescribed was ferrous sulphate.

Darboepoetin alfa, a second generation erythropoiesisstimulating agent, contains 2 amino acid substitutions, which provide the protein with greater metabolic stability in vivo and increase the elimination half-life when compared with intravenous epoetin alfa (25.3 hours vs 8.5 hours for epoetin alfa) was invented. A large randomized, double-blind multicenter study consucted in over 500 hemodialysis patients demonstrated that once-weekly darbepoetin alfa was as effective in maintaining hemoglobin levels as epoetin dosed 3 times weekly for the treatment of anemia. A secondary analysis of this study was conducted to determine whether the type of erythropoietic agent that is used might also influ- ence hemoglobin variability in hemodialysis patients which showed that there was no clinically or statistically significant difference in median within subject hemoglobin variance between darbepoetin alfa-treated and epoetin alfa treated subjects .i.e. hemoglobin variability in this study population was not influenced by the type of erythropoietic agent used for the treatment of anemia. ${ }^{8}$

Our study also depicts the attempts made to monitor hypertension in the chronic kidney disease patients and hence in our study statistically significant decrease in the systolic blood pressure was observed for pre-hemodialysis and for post hemodialysis.

Elke Wuhl has stated that efficient control of blood pressure and minimization of proteinuria appears as the two most important measures to preserve residual kidney function. ${ }^{9}$

Patient counseling was given based on medication adherence, adherence to prescribed diet and fluid intake.

In our counseling on medication adherence we counseled the patients on the following aspects;

- To store medications in a clean, safe, dry place away from heat, light and moisture- and out of reach of children.

- To learn the names and uses of any medications prescribed for them.

- To inform the doctor about all medications being taken i.e. both prescription and non-prescription which is to be administered for chronic kidney disease or any other conditions (to prevent harmful drug interactions).

- To inform the doctor of any drug allergies.

- To keep a record of when to take the medicationespecially in case of polypharmacy.

- Not to substitute one type of medication for another without the doctor's approval.

- Not to skip doses or double a dose when one has been missed.

- To keep the blood sugar, blood pressure, and blood cholesterol within the target range.

- To take their diet as prescribed by the dietitian.

- To take fluid as prescribed by the doctor.

In general, CKD patients were asked to reduce on salt and excessive protein (not allowed to take organ meat as well as red meat), and control blood sugar if they were diabetic. The patient information leaflets were prepared and then given to each patient to keep them informed on the CKD and its causes, anemia, causes of anemia, signs and symptoms of anemia, complications of anemia, benefits of management of anemia in CKD and how to control anemia and thereby keep their kidneys healthy. 
Heightened risk for progression of kidney failure has also been linked to untreated anemia of CKD. Thus, management of anemia throughout the CKD continuum is essential.

Pharmacists are very good at helping patients obtain therapeutic goals or outcomes by being vigilant about the pharmacotherapy that is employed with the CKD patients and hence pharmacists definitely have a role in anemia management in CKD patients. ${ }^{10}$

\section{CONCLUSION}

Early detection and aggressive treatment of anemia in CKD patients is very essential to improve patient's health related quality of life. It is always a challenge to develop modules for integrating care giver community with adequate patient information and treatment for improved patient care.

\section{ACKNOWLEDGEMENT}

We express our deep sense of gratitude to the staff, Department of Pharmacy Practice, Visveswarapura Institute of pharmaceutical Sciences, Bengaluru. The authors would like to thank the doctors and the working staff of dialysis department, KIMS Bengaluru. We also thank all the patients for consenting to take part in our study and giving the relevant information which were of great significance for completion of the study.

\section{CONFLICT OF INTEREST}

None

\section{REFERENCES}

1. MacGinley R, Walker R. International treatment guidelines for anaemia in chronic kidney disease -what has changed?. Med J Aust. 2013;199(2):84-5. http://dx.doi.org/10.5694/mja13.10538; PMid:23879487.

2. Padhi S, Glen J, Pordes B, Thomas M. Management of anaemia in chronic kidney disease: summary of updated NICE guidance. BMJ. 2015;350(jun04 6): h2258-h2258.

3. Willis K, Cheung M, Slifer S. Kidney International Supplements. Kidney Int. 2012;2(4):292-8.

4. Schmidt R, Dalton $C$. Treating anemia of chronic kidney disease in the primary care setting: cardiovascular outcomes and management recommendations. Osteopathic Medicine and Primary Care. 2007;1(1):14. http://dx.doi. org/10.1186/1750-4732-1-14 ; PMid:17910755 PMCid:PMC2147011.

5. Singh A, Coyne D, Shapiro W, Rizkala A. Predictors of the response to treatment in anemic hemodialysis patients with high serum ferritin and low transferrin saturation. Kidney Int. 2007;71(11):1163-71.http://dx.doi. org/10.1038/sj.ki.5002223 ; PMid:17396118.

6. Hörl W. Iron therapy for renal anemia: how much needed, how much harmful?. Pediatr Nephrol. 2007;22(4):480-9. http://dx.doi.org/10.1007/s00467-0060405-y ; PMid:17206511 PMCid:PMC1805051.

7. Del Vecchio L, Cavalli A, Pozzoni P, Locatelli F. Recombinant human epoetin beta in the treatment of renal anemia. There Clin Risk Manage. 2007;3(3):433-439.

8. Fricke K. Treatment of Anemia in Chronic Kidney Disease: Past Present, Future. Medscape Nephrology. 2006.

9. Wühl E, Schaefer F. Therapeutic strategies to slow chronic kidney disease progression. Pediatr Nephrol. 2008;23(5):705-16. http://dx.doi.org/10.1007/ s00467-008-0789-y ; PMid:18335252 PMCid:PMC2275772.

10. Grabe DW, Manley HJ, Pai AB. Advances in Chronic Kidney Disease. Management of Anemia and the Role of Pharmacy. 2007. 